\title{
Context of MUC1 epitope: Immunogenicity
}

\author{
I.S. QUINLIN ${ }^{1,2,3,5}$, J.S. BURNSIDE ${ }^{1,3}$, K.E. DOMBROWSKI ${ }^{1,3,6,7}$,

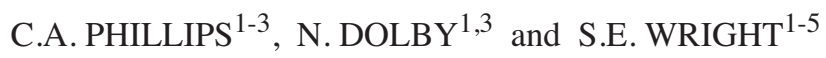 \\ ${ }^{1}$ Amarillo Veterans Affairs Medical Center, 6010 Amarillo Blvd. West; ${ }^{2}$ Women's' Health Research Institute, \\ Departments of ${ }^{3}$ Internal Medicine, School of Medicine, ${ }^{4}$ Pharmaceutical Sciences, School of Pharmacy, Texas \\ Tech University Health Sciences Center, 1400 Wallace Blvd.; ${ }^{5}$ Harrington Cancer Center, 1500 Wallace Blvd., \\ Amarillo, TX 79106; ${ }^{6}$ The Atlanta Research and Education Foundation, Decatur, GA 30033; ${ }^{7}$ Department of \\ Microbiology and Immunology, Emory University School of Medicine, Atlanta, GA 30322, USA
}

Received September 27, 2006; Accepted October 30, 2006

\begin{abstract}
MUC1 is a glycoprotein found at the secretory poles of normal cells but is hypoglycosylated on the entire surface of cell membranes of adenocarcinomas. In order to determine the influence on the immune response of peptide context for epitope presentation, peripheral blood mononuclear cells (PBMC) from patients with adenocarcinomas, were stimulated with MUC1 peptides derived from the 20 amino acids (aa) long sequence that is characteristic of the MUC1 Variable Number of Tandem Repeats (VNTR). In the seven peptides tested, the T-cell tumor-specific epitope (cTSE) was surrounded by variable numbers of aa and repeated up to 5 times in the same peptide. The results of this study indicate that cultures stimulated with peptide 610 (GSTAPPAHGVTS APDTRPAP) showed the highest specific killing of the MUC1expressing breast cancer MCF-7 cells. Peptide 610 is also superior to the other peptides in inducing better production of the type 1 cytokines, tissue necrosis factor $\alpha$ and interferon $\gamma$. In conclusion, context of the epitope and not sequence alone determines immunogenicity.
\end{abstract}

\section{Introduction}

MUC1 is one of the glycoproteins of mucus secretions. The core of the MUC1 protein is usually coated heavily with carbohydrates; i.e., glycosylated. The mass of MUC1 (80\%) is made up of carbohydrates. The extensive branching for these carbohydrate side chains inhibits the tumor-specific antigenicity and immunogenicity of normal MUC1. However, shorter carbohydrates are produced on MUC1 of many cancer cells, exposing epitopes on the core protein to the immune

Correspondence to: Dr Stephen E. Wright, Amarillo Veterans Affairs Medical Center, 6010 Amarillo Blvd. West, Amarillo, TX 79106, USA

E-mail: stephen.wright@med.va.gov

Key words: context, mucin 1, immunogenicity system (1). These aberrantly glycosylated cancer-associated MUC1 are immunogenic and are abundantly expressed by breast carcinomas as well as other carcinomas such as pancreatic, ovarian, lung, renal, colon, gastric, prostate and urinary bladder (2).

The entire coding sequence for the core protein of MUC1 has been cloned and its amino acid sequence determined (3). The core of the MUC1 protein consists of three regions: the extracellular region containing the VNTRs, the transmembrane domain and the cytoplasmic tail. A general characteristic of the MUC1 core proteins is tandem repeats. Each repeat consists of an identical 20 amino acid sequence (PDTRPAPGSTAPP AHGVTSA). Different numbers of tandem repeats are the cause of the polymorphism exhibited by MUC1.

Initial experiments showed that unglycosylated MUC1 contains a tumor specific epitope. This epitope was detected with the monoclonal antibody, SM3, which recognized tumorspecific MUC1 and fully deglycosylated native MUC1 but not the fully glycosylated form and thus did not react with normal tissues (4). By inserting one VNTR MUC1 tandem repeat into an $E$. coli expression vector, we were able to produce an unglycosylated maltose-binding protein (MBP)MUC1 fusion protein. These proteins were purified and showed strong affinity to tumor-specific monoclonal antibody SM3 (5). Competition assays of SM3 with anti-MBP-MUC1 showed that the SM3 bound the same epitope on the MBP-MUC1 fusion protein as the anti-MBP-MUC1 (5). These experiments helped substantiate the hypothesis that unglycosylated MUC1 contains a tumor-specific epitope and opened the possibility of using MUC1 in developing vaccines against adenocarcinomas and cytotoxic T-lymphocyte (CTL) lines for immunotherapy of adenocarcinomas. The human MUC1 monoclonal antibody tumor-specific epitope (MAB-TSE) is found within the pentapeptide PDTRP sequence present in the MUC1 VNTR motif (4).

Barnd et al (6) and Ioannides et al (7) have speculated that the likely T-cell tumor-specific epitope (cTSE) is included in, or adjacent to, the same epitope that is recognized by the SM3 monoclonal antibody. Blocking CTL cytotoxicity of MCF-7 with SM3 monoclonal antibody narrowed the CTL epitope to 10 aa (P1 DTRPAPGST10). Antibodies recognizing epitopes outside this 10 aa sequence had no inhibitory effect 


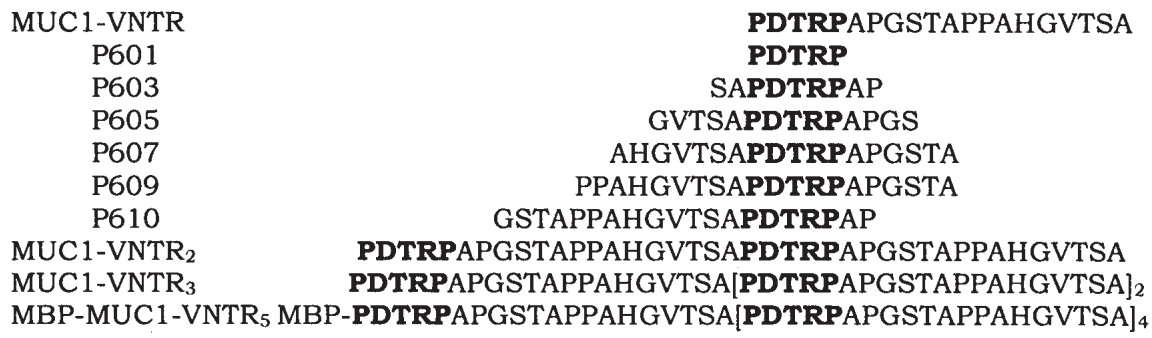

Figure 1. MUC1 cTSE in various contexts of the MUC1 20 aa VNTR motif peptides in various contexts.

on MUC1-carcinoma-specific CTL killing of MUC1-producing adenocarcinomas. This suggests that the SM3-binding site (MAB-TSE) and cTSE are the same or near one another. However, it was not clear whether the inhibition of cytolytic activity with SM3 was due to steric hindrance as a result of its binding in the proximity of the cTSE or directly to it. Using human leukocyte antigen (HLA)-2 transgenic mice, Apostolopoulos et al found a cTSE (STAPPAHGV), at the opposite end of the MUC1 VNTR motif (8). This cTSE is present in the class II MHC epitope PGSTAPPAHGVT (9). The study presented addressed the context of the cTSE(s), i.e., the orientation or location within the VNTR, and immunogenicity.

\section{Materials and methods}

MUC1 peptides (Fig. 1). MUC1 VNTR peptides were synthesized by American Peptide Co., Inc., Sunnyvale, CA and Chiron, Emeryville, CA, USA (P601, P603, P605, P607, P609 and P610). MBP-VNTR5 was produced in our laboratory (10).

Cell culture conditions. PBMC were from humans with breast adenocarcinomas from apheresis, in accordance with the institutional review board. Cells were cultured at $2 \times 10^{6}$ cells $/ \mathrm{ml}$ in AIM-V (Registered ${ }^{\mathrm{TM}}$ ) serum-free lymphocyte medium (Life Technologies Gibco-BRL, Grand Island, NY, USA) and maintained in a $37^{\circ} \mathrm{C}$ humidified $5 \% \mathrm{CO}_{2}$ atmosphere. IL-2 (Cetus) was added twice per week at $100 \mathrm{IU} / \mathrm{ml}$. Cells were stimulated with $1 \mu \mathrm{g} / \mathrm{ml}$ MUC1-VNTR1 peptide on days 0 and 7 . Media was not changed.

Cytotoxicity assays. MCF-7 breast cancer cell line was obtained from, and cultured as recommended, by the American Type Culture Collection (ATCC). MCF-7 expresses hypoglycosylated MUC1 (6). This cell line was used as the target cell line in a ${ }^{51} \mathrm{Cr}$ release assay (11). The target cells were labeled with $\left[{ }^{51} \mathrm{Cr}\right]$ sodium chromate (New England Nuclear) $(200 \mu \mathrm{Ci}$ per $1 \times 10^{7}$ cells) and $5 \times 10^{3}$ target cells/well of microtiter plates were added. The effector cells were tested at the effector to target cell ratios (E:T) indicated in Table I. Medium was added in place of effector cells to the control wells measuring the spontaneous ${ }^{51} \mathrm{Cr}$ release. In maximum target lysis control wells, effector cells were replaced with 2 or $10 \%$ Triton X-100 (Sigma). The assays were incubated for $18 \mathrm{~h}$ at $37^{\circ} \mathrm{C}$ and $5 \%$ $\mathrm{CO}_{2}$, and performed in triplicate. The cells were centrifuged and 50 of the $100 \mu 1$ supernatants were harvested. Radioactivity
Table I. Killing of MUC1 expressing adenocarcinoma cells with MUC1 peptides stimulated PBMC.

Day 17

STIM $^{\text {a }}$ a.011597 $\quad$ b.020696 c. 073093 d. 367969-2

\begin{tabular}{lcllc}
\hline IL-2 & 4 & 16 & 26 & 13 \\
601 & ND $^{\mathrm{c}}$ & ND & 22 & 10 \\
603 & ND & ND & 45 & 7 \\
605 & ND & 28 & 44 & 12 \\
607 & ND & 18 & 23 & 11 \\
609 & 54 & 34 & 22 & 13 \\
610 & ND & 48 & 57 & $18^{\text {d }}$ \\
VNTR $_{1}$ & 14 & 26 & ND & 6 \\
VNTR $_{2}$ & 15 & ND & ND & ND \\
VNTR $_{3}$ & 39 & ND & 42 & ND \\
MBP- $^{-}$ & & & & \\
VNTR $_{5}$ & ND & 27 & 38 & ND \\
MBP $^{\text {NNST }}$ & ND & 21 & ND & ND \\
UNST $^{\text {b }}$ & 3 & 0 & 0 & ND \\
\hline
\end{tabular}

Percent specific lysis of MCF-7 at E:T=10:1 by PBMC from patients with breast cancer after stimulations on days 0 and 7 , and incubation for one month. aStim, stimulant, with IL-2 twice per week as in Materials and methods. bunst, unstimulated. Frozen on the day of preparation of PBMC. Thawed the day before the assay and placed into media without IL-2 and assayed on the day of the cytotoxicity assay. ${ }^{\mathrm{C} N D}$, not performed. ${ }^{\mathrm{d} P} 610 \mathrm{E}: \mathrm{T}: 1.25: 1,2.5: 1,5: 1 . \%$ specific lysis: $8,10,13$. Other peptides-stimulated PBMC lysed $\leq 12 \%$ $\mathrm{MCF}-7$ at the lower $3 \mathrm{E}: \mathrm{T}$ ratios.

released into the supernatant was measured by liquid scintillation or $\gamma$ counting. The specific lysis was calculated by:

$\%$ lysis $=\frac{(\text { mean experimental } \mathrm{cpm}-\text { mean spontaneous } \mathrm{cpm})}{(\text { mean maximum } \mathrm{cpm}-\text { mean spontaneous } \mathrm{cpm})} \times 100$

Cytokine assays. Assays for cytokines (interferon $\gamma$ (INF- $\gamma$ ) and tumor necrosis factor $\alpha$ (TNF- $\alpha$ ) (BD Pharmingen Inc., San Diego, CA, USA), were performed by the use of enzyme linked solid phase sandwich assay (ELISA) kits per manufacturer's instructions. The detection limit was $6 \mathrm{pg} / \mathrm{ml}$. 

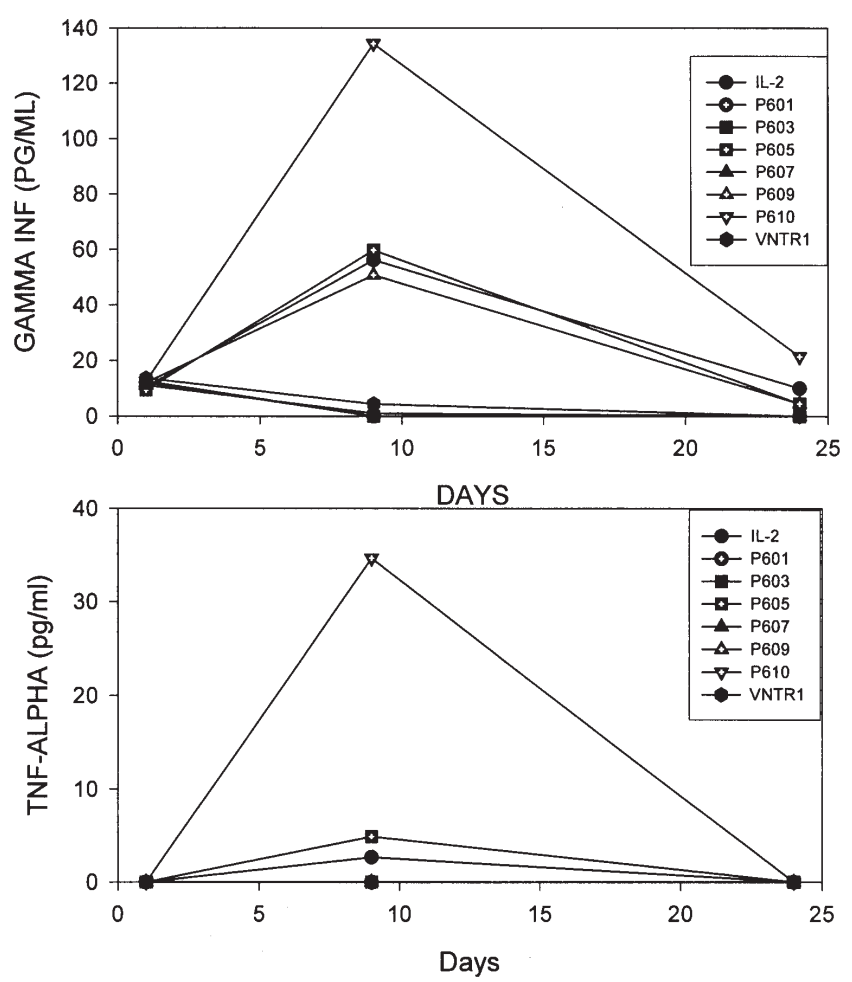

Figure 2. Cytokine production from MUC1 peptides stimulated PBMC. Interferon $\gamma$ (top) and tissue necrosis factor $\alpha$ (bottom) produced by PBMC 367969 , from a patient with breast cancer, stimulated on days 0 and 7 with MUC1 peptides, as determined by ELISA assays. Supernatant was collected on days 1,8 , and 24. Data points overlap at baseline levels.

Supernatant from stimulated PBMC were added to the cytokine monoclonal antibody coated 96-well plates and incubated. The wells were then emptied and washed. Streptavidinhorseradish peroxidase conjugate mixed with a biotinylated anti-human antibody for the specific cytokine was added. The wells were washed. The substrate solution $\left(3,3^{\prime}, 5,5^{\prime}\right.$ tetramethylbenzidine) was added. After $30 \mathrm{~min}$, the stop solution (1 M phosphoric acid) was added. The wells were read at $450 \mathrm{~nm}$. A set of standards with known amounts of cytokine were run with each assay to develop a standard curve. The amount of cytokine present in the samples was determined using this standard curve.

\section{Results}

Cytotoxicity. To attempt to identify the cTSE and optimal context, PBMC preparations from four patients with breast cancer were stimulated with peptides from Fig. 1 (Table I). Of the seven VNTR peptides of $\leq 20$ aa, peptide P610-, a 20 mer with PDTRP 2 aa from the carboxy terminus, stimulated lymphocytes having the greatest toxicity against MCF7. This implies that PDTRP near the carboxy terminus may enhance immunogenicity of the VNTR. MBP-VNTR ${ }_{5}(10)$ was no better than the optimum $\mathrm{VNTR}_{3}$, implying that three repeats may be the maximum needed to stimulate CTL in the (PDTR PAPGSTAPPAHGVTSA) orientation.

Cytokine production. Type 1 cytokines are produced by antigen-activated lymphocytes, thus supernatant samples taken
Table II. Production of INF- $\gamma$ by PBMC from a patient with breast cancer stimulated with MUC1 peptides.

IL-2 P601 P603 P605 P607 P609 P610 VNTR1

\begin{tabular}{lrrrrrrrr}
\hline Mean $^{\mathrm{a}}$ & 34 & 0 & 0 & 37 & 0 & 31 & 86 & 1 \\
$\mathrm{SD}^{\mathrm{b}}$ & 2 & 0 & 0 & 1 & 0 & 3 & 2 & 1 \\
\hline
\end{tabular}

PBMC 367969-2 stimulation with MUC1 peptides was on days 0 and 7. Supernatant of MUC1 peptides stimulated PBMC was assayed by ELISA on day 8 . ${ }^{\mathrm{a}} \mathrm{pg} / \mathrm{ml}$. ${ }^{\mathrm{b}} \mathrm{SD}$, standard deviation, $\mathrm{N}=3$. Only the PBMC stimulated with P610 produced a statistically different ( $p<0.05$, pair-wise multiple comparisons of the eight conditions by the Student-Newman-Keuls method) amount of INF- $\gamma$, compared to other peptide contexts.

Table III. Production of TNF- $\alpha$ by PBMC from a patient with breast cancer stimulated with MUC1 peptides.

\section{IL-2 P601 P603 P605 P607 P609 P610 VNTR1}

\begin{tabular}{lllllllrl}
\hline Mean $^{\mathrm{a}}$ & 3 & 0 & 0 & 5 & 0 & 0 & 35 & 0 \\
$\mathrm{SD}^{\mathrm{b}}$ & 1 & 0 & 0 & 1 & 0 & 0 & 3 & 0
\end{tabular}

PBMC 367969-2 stimulation with MUC1 peptides was on days 0 and 7. Supernatant of MUC1 peptides stimulated PBMC was assayed by ELISA on day 8. ${ }^{\mathrm{a}} \mathrm{pg} / \mathrm{ml}$. ${ }^{\mathrm{b}} \mathrm{SD}$, standard deviation, $\mathrm{N}=3$. Only the PBMC stimulated with P610 produced a statistically different ( $p<0.05$, pair wise multiple comparisons of the eight conditions by the Student-Newman-Keuls method) amount of TNF- $\alpha$, compared to other peptide contexts.

on days 1,8 and 24 were tested for the cytokines TNF- $\alpha$, INF- $\gamma$, IL-10 and GM-CSF by ELISA. There was not a detectable amount of Type 2 cytokine, IL-10 or GM-CSF in any of the supernatant samples from this experiment. Type 1 cytokines, INF- $\gamma$ and TNF- $\alpha$, however, were produced by PBMC stimulated with some of the MUC1 peptides. The maximum production of both of these cytokines occurred on day 8 [there are overlapping data points at baseline (Fig. 2)].

On day 8, only PBMC stimulated with the MUC1 peptide P610 produced INF- $\gamma$ significantly greater than PBMC stimulated with IL-2. PBMC stimulated with the other MUC1 peptides produced similar or less INF- $\gamma$ on day 8 than by cells stimulated with IL-2 (Table II). Pair-wise multiple comparisons of the eight MUC1 peptides were made using the Student-Newman-Keuls method. Only the PBMC stimulated with P610 produced a statistically different amount of INF- $\gamma(\mathrm{p}<0.05)$.

In addition, only PBMC stimulated with the MUC1 peptide P610 produced TNF- $\alpha$ on day 8 significantly greater than the detection limit of $6 \mathrm{pg} / \mathrm{ml}$ (Table III). The PBMC stimulated with P610 were the only PBMC to produce a statistically different amount of TNF- $\alpha(\mathrm{p}<0.05)$. 


\section{Discussion}

The purpose of the experiments was to determine if the aa surrounding the MAB-TSE influences the cellular immune response. The DTR epitope is a well-known MAB target to several tumor-specific MAB, including SM3 (4) and HMFG2 (12). Therefore, to attempt to identify the CTL epitope, seven MUC1 peptides (MUC1-VNTR 1 , P601, P603, P605, P607, P609 and P610), with different epitope contexts and different number of repeats $\left(\mathrm{VNTR}_{1,3,5}\right)$, were used to stimulate PBMC (Fig. 1). The shortest peptide, P601, contained only the MABTSE ( $\underline{\text { PDTRP) }}$. Previous studies have suggested that placement of the epitope near the carboxy terminus of a peptide is more immunogenic $(13,14)$. Two peptides, P603 (SAPDTRPAP) and P610 (GSTAPPAHGVTSAPDTRPAP) had only two aa following the epitope on the carboxy terminus. On the other

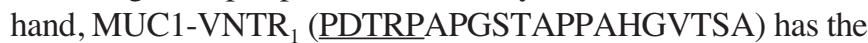
epitope at the amino terminus. In P605 (GVTSAPDTRPAPGS) the MAB-TSE is 4 aa away from the carboxy terminus. The epitope is 6 aa away from the carboxy terminus in P607 (AH GVTSAPDTRPAPGSTA) and P609 (PPAHGVTSAPDTRP APGSTA). VNTR $2,3,5$ have one or more of the DTR epitope at non-terminal locations.

In the study presented herein, PBMC obtained from patients with breast cancer were stimulated with seven different peptide contexts of MUC1 VNTR, and different numbers of VNTR. $\mathrm{VNTR}_{3}$ was the optimum number of VNTR. VNTR 3 may assume secondary structure, and if so, implies that structure is sufficient for immunogenicity. The other finding was that PBMC stimulated with the MUC1 peptide GSTAPPAHGVTSAPDTRPAP (P610) exhibited the highest specific lysis of the MCF-7 and produced a greater amount of the type 1 cytokines INF- $\gamma$ and TNF- $\alpha$ than PBMC stimulated with other MUC1 peptides. The enhanced killing of MCF-7 cells by PBMC stimulated with the MUC1 peptide GSTAPPAHGVTSAPDTRPAP (P610) is possibly due to the context of the PDTRP epitope, near the carboxy terminus, which has been shown to enhance immunogenicity with other antigens $(13,14)$. However, a PDTRP as a 9 (P603), 14 (P605), 18 (P607) or 20 (P609) mer does not appear to be as immunogenic as 20 mer P610 which contains STAPPAHGV, supporting the data $(8,9)$ for STAPPAHGV as the cTSE epitope(s). If STAPPAHGV (8) and/or PGSTAPPA HGVT (9) are the critical cTSE epitope(s), then the opposite context, with cTSE epitope(s) near the amino terminus may be more immunogenic. P610 is the only peptide containing STAPPAHGV at the amino terminus. VNTR $_{1}$ contains STAPPAHGV, but at the carboxy end. In conclusion, context of the epitope and not sequence alone determines immunogenicity.

\section{Acknowledgements}

This study was performed in partial fulfillment of the requirements of the degree Masters of Science from West Texas A\&M University, Canyon, TX, USA. The authors are grateful to Glenda Ramsey, Baptist-St. Anthony's HSC, Harrington Cancer Center Cell Processing Laboratory, Amarillo, TX for PBMC, and to Crystal Tabor and Kyndness Garza for editorial assistance. This study was supported in part by the Department of Veteran's Affairs Medical Research funds (S.E.W.; K.E.D. and S.E.W.), the Department of the Army Career Develop-ment Award grant DAMD17-94-J-4161 (K.E.D.) and the Department of the Army Grant DAMD1794-J-4272 (K.E.D. and S.E.W.).

\section{References}

1. Gendler SJ: MUC1, the renaissance molecule. J Mammary Gland Biol Neoplasia 3: 339-353, 2001.

2. Devine P, McGuckin M, Ramm L, Ward B, Pee D and Long S: Serum mucin antigens CASA and MSA in tumors of the breast, ovary, lung, pancreas, bladder, colon and prostate: a blind trial with 420 patients. Cancer 72: 2007-2015, 1993.

3. Gendler SJ, Lancaster CA, Taylor-Papadimitriou JT, Duhig T, Peat N, Burchell J, Pember-ton L, Lalani EN and Wilson D: Molecular cloning and expression of human tumor associated polymorphic epithelial mucin. J Biol Chem 265: 15286-15293, 1990

4. Gendler SJ, Spicer AP, Lalani EN, Duhig T, Peat N, Burchell J, Pemberton L, Boshell M and Taylor-Papadimitriou J: Structure and biology of a carcinoma-associated mucin, MUC1. Am Rev Respir Dis 144: S42-S47, 1991.

5. Hu P and Wright SE: Expression of a recombinant breast tumorassociated mucin fusion protein in Escherichia coli exposes the tumor-specific epitope. Cancer Res 53: 4920-4926, 1993.

6. Barnd DL, Lan MS, Metzgar RS and Finn OJ: Specific, major histocompatibility complex-unrestricted recognition of tumorassociated mucins by human cytotoxic T cells. Proc Natl Acad Sci USA 86: 7159-7163, 1989.

7. Ioannides CG, Fisk B, Jerome KR, Irimura T, Wharton JT and Finn OJ: Cytotoxic $\mathrm{T}$ cells from ovarian malignant tumors can recognize polymorphic epithelial mucin core peptides. J Immunol 151: 3693-3703, 1993.

8. Apostolopoulos V, Karanikas V, Haurum JS and McKenzie I: Induction of HLA-A2-restricted CTLs to the mucin 1 human breast cancer antigen. J Immunol 159: 5211-5219, 1997.

9. Hiltbold EM, Ciborowski P and Finn OJ: Naturally processed class II epitope from the tumor antigen MUC1 primes human $\mathrm{CD}^{+} \mathrm{T}$ cells. Cancer Res 58: 5066-5070, 1998.

10. Dolby N, Dombrowski KE and Wright SE: Design and expression of a synthetic mucin gene fragment in Escherichia coli. Protein Expr Purif 15: 146-154, 1999.

11. Jin Y, Giri C, Klutch MJ, Shepp D and Wright SE: Improved immunogenicity of recombinant vaccinia virus-anchored gp120 lacking gp41. Vaccine 11: 1280-1282, 1993.

12. Magarian-Blander J, Domenech N and Finn OJ: Specific and effective T-cell recognition of cells transfected with a truncated human mucin cDNA. Ann NY Acad Sci 690: 231-243, 1993.

13. Golvano J, Lasarte JJ, Sarobe P, Gullon A, Prieto J and BorrasCuesta F: Polarity of immunogens: implications for vaccine design. Eur J Immunol 20: 2363-2366, 1990.

14. Stevenson FK, Ottensmeier CH, Johnson P, Zhu D, Buchan SL, McCann KJ, Roddick JS, King AT, McNicholl F, Savelyeva N and Rice J: DNA vaccines to attack cancer. Proc Natl Acad Sci USA 101: 14646-14652, 2004. 